\title{
POSSÍVEIS EFEITOS ALELOPATICOS DE Brachiaria decumbens SOBRE O CRESCIMENTO INICIAL DE LIMÃO CRAVO (Citrus limonia) ${ }^{1}$
}

\author{
SOUZA L. S. ${ }^{2}$, VELINI, E. D. ${ }^{3}$, MARTINS, D. ${ }^{3}$, ROSOLEM, C. A. ${ }^{4}$
}

\section{RESUMO}

Foram estudados os possíveis efeitos alelopáticos da incorporação da matéria seca da parte aérea de Brachiaria decumbens, ao substrato, nas concentrações de $0,1,5$ e 3,0\% (p/p) sobre o crescimento inicial de limão cravo (Citrus limonia Osbeck), em casa-de-vegetação. Foram de te rminad os os parâmetros bi omét ricos e algumas propriedades físico-químicas da solução do solo. A maior quantidade de matéria seca incorporada causou redução de 44, 42, 57 e 55\% das médias da altura, teor de clorofila, área foliar e acúmulo de matéria seca total, respectivamente. $\mathrm{O}$ potencial osmótico, $\mathrm{pH}$, condutividade elétrica e os teores de nutrientes da solução do solo foram alterados. Provavelmente, a redução do crescimento observada seja devida a deficiência de $\mathrm{N}$ causada por alterações na dinâmica do $\mathrm{N}$-solo em função da incorporação de $B$. decumbens.

Palavras chave: Alelopatia, nutrientes, solução de solo.

\section{ABSTRACT \\ The possible allelopathic effects of Brachiaria decumbens on initial development of root stock of Citrus limonia.}

It was studied the possible allelopathic effect of Brachiaria decumbens on initial development of Citrus limonia in greenhouse. The dry matter was incorporated into the soil in $0, \quad 1,5, \quad 3,0 \% \quad(\mathrm{w} / \mathrm{w})$-proportion. Biometric parameters and physic properties of soil were determined by soil solution. Greatest propotion reduced all the biometric parameters analysed. Physical properties of nutrient levels (except $\mathrm{N}$ ) of soil did not observed significantly alterations. Greatest proportion reduced the average stem diameter of Citrus limonia at 57\%. Probably, these results were due to lack in nitrogen level observed in soil solution.

Key words: Allelopathy, nutrient, soil solution.

1Recebido para publicação em 22/08/97 e na forma revisada em 15/12/97.

2Eng. Agr. M. Sc., Aluno do Curso de Pós-Graduação em Agricultura-FCA/UNESP, (Bolsista da CAPES).

3 Prof. Assistente Doutor. Depto. Agricultura e Melhoramento Vegetal, FCA/UNESP, Caix a Postal 237, CEP 18603 -970, Botucatu - SP.

3Prof. Assistente Doutor, Depto. Agricultura e Melhoramento Vegetal, FCA/UNESP.

4 Prof. Titular, Depto. Agricultura e Melhoramento Vegetal, FCA/UNESP. 


\section{INTRODUÇÃO}

Na região de Botucatu (São Paulo-Brasil) são cultivados, atual mente, 3.317.703 pés de várias espécies cítricolas, que têm assumido um papel de grande importância econômica na região nas últimas décadas (Sorocaba, 1997).

A agropecuária "Companhia Agrícola de Botucatu", localizada nos municípios de Botucatu e Itatinga - SP, possui uma área com 400.000 pés de laranja e 300.000 pés de limão, dentre os quais, 550.000 pés foram enxertados sobre limão cravo (Citrus limonia).

Nessa agropecuária, Almeida* constatou uma redução na altura das mudas enxertadas com limão siciliano (Citrus limon) plantadas em antigas pastagens de $B$. decumbens. Tal fato não ocorreu em mudas dessa espécie, na mesma linha de plantio, porém plantadas em antigas pastagens de setária (Secaria geniculata) (Comunicação verbal). Na mesma região de Botucatu - SP, Souza (1994) demonstrou efeitos inibitórios da $B$. decumbens sobre o desenvolvimento inicial de mudas de Eucaliptus grandis, decorrentes da decomposição da matéria seca dessa espécie de planta daninha. Com base nessas informações, pode-se especular a ocorrência de possíveis efe itos ale lopáticos da $B$. decumbens sobre as mudas de limão siciliano, impla ntadas nas áreas de pastagens da "Companhi a Agrícola de Botucatu".

Sabe-se que durante a decomposição microbiana, vários compostos químicos são formados e dissolvidos na solução do solo, onde es se s de sempen ham imp or tante ativida de biológica. Foram identificados, nestas condições, vários al eloquímicos, como sendo produtos resultantes da decomposição de plantas daninhas, as sociad os aos mic roorgan is mos do solo, que atuam na inibição de nutrientes disponíveis para a planta (Olmsted \& Rice, 1970; Buchholtz, 1971). De acordo com Rice (1984), somente as fases do

\footnotetext{
* Almeida, E.L.P., Eng. Agr. (Gerente da Compahia Agricola de Botucatu-Botucatu-SP) Comunicação Verbal, 1997.
}

ciclo do nitrogênio que envolvem plantas ou microrganismos é que podem ser afetadas pela alel opatia, segundo a definição de Molisch. Por outro lado, a própria adição de matéria orgânica pode afetar o ciclo do nitrogênio.

Há consideráveis evidências na literatura atribuindo efeitos alelopáticos da matéria orgânica sobre a fixação de nitrogênio, mas existem poucos estudos relacionando o efeito alelopático direto e a quantidade de matéria orgânica adicionada ao solo (Rice, 1984).

O presente estudo te ve por objetivo determinar os possíveis efeitos alelopáticos de diferentes quantidades de matéria seca da parte aérea de $B$. decumbens incorporadas ao substrato sobre o crescimento inicial de limão cravo.

\section{MATERIAL E MÉTODOS}

O experimento foi conduzido em casa-devegetação, na Faculdade de Ciências Agronômicas/UNESP/Câmpus de Botucatu - SP. A terra utilizada como substrato no experimento foi coletada da camada arável de um Latossolo Roxo. A terra, de pois de coletada, foi seca à sombra e peneirada (malha de $5 \mathrm{~mm}$ ).

Foram utilizados vasos plásticos com capacidade para 7 litros. Visando evitar eventuais perdas de água por percolação durante o processo de irrigação, foram utilizados vasos com base sem drenos.

As mudas de porta-enxerto de limão cravo, na fase de transplante, foram fornecidas pela Companhia Agrícola de Botucatu.

Plantas de B. decumbens foram coletadas na Fazenda Morrinhos, pertencente a Companhia Agrícola de Botucatu. A parte aérea foi seca em estufa de circulação forçada de ar, a $60^{\circ} \mathrm{C}$ por 72 horas e, a seguir, triturada em moinho (malha de $0,7 \mathrm{~mm}$ ) e armazenada em câmara seca.

O substrato de crescimento das mudas foi corrigido através da análise físico-química da terra, que esta apresentada na Tabela 1. Foram adicionadas as seguintes quantidades de nutrientes: $\mathrm{N}=150 \mathrm{ppm}$ (úreia), $\mathrm{P}=200 \mathrm{ppm}$ (superfosfato simples) e $\mathrm{K}=150 \mathrm{ppm}$ (cloreto de 
potássio). Foram utilizad os um mist urad or giratório manual para incorporação homogênea dos nutrientes e da matéri a seca da planta daninha ao substrato. A parte aérea da planta da ninha foi incor porada ao substrato nas con centrações de 0 (te ste munha), 1,5 e 3,0\% $(\mathrm{p} / \mathrm{p})$. Cada vaso continha $5 \mathrm{~kg}$ de terra como substrato.

Tabela 1. Características físico-químicas da terra utilizada como substrato nos experimentos. Botucatu SP, 1995.

\begin{tabular}{cccccccccc}
\hline M.O. & $\mathbf{p H}$ & $\mathbf{P}$ & \multicolumn{7}{c}{$\mathbf{m e g} / \mathbf{1 0 0 \mathbf { c m } ^ { 3 }}$} \\
\cline { 5 - 9 }$\%$ & $\left(\mathrm{CaCl}^{2}\right)$ & $\left(\mu \mathbf{g P} / \mathbf{c m}^{3}\right)$ & $\mathbf{K}^{+}$ & $\mathbf{C a}^{++}$ & $\mathbf{M g}^{++}$ & $\left(\mathbf{H}^{++} \mathbf{A l}^{+\mathbf{3}}\right)$ & $\mathbf{S}$ & $\mathbf{C T C}$ & $\mathbf{V ~ ( \% )}$ \\
\hline 2,60 & 5,50 & $6,0^{\mathrm{a}}$ & 0,17 & 1,7 & 1,0 & 2,2 & 2,9 & 5,1 & 56 \\
\hline
\end{tabular}

(a) método da resina (Rai) \& Quaggio, 1983)

Em seguida, foi realiza do o transplante das mudas de limão para os vasos. Em cada vaso foi instalado um coletor de solução de solo com cápsula de porcelana porosa (Moraes \& Dynia, 1990 ). Para a retira da da solução de solo acumulada nos coletores, ao longo do experimento, foi utilizada uma bomba de vácuo manual acoplada em uma das mangueiras da cápsula porosa (Hossner \& Phillips, 1973). A irrigação foi realizada para manter a umidade da $70 \%$ da capacidade de saturação do substrato.

O experimento foi conduzido até 58 dias após o trans plante das mudas. Foram avaliados, em cada muda, os sequintes aspectos: altura das plantas, diâmetro do caule, número de folhas, teor de clorofila total nas folhas e biomassa seca das raízes, caules e folhas. Para a avaliação do teor de cl or of ila total, foi utilizado um medidor automático de clorofila (clorofilômetro) marca Minolta, modelo SPAD 502.

Ao final do experimento, a parte aérea das mudas foi coletada, separando-se as folhas do caule, e medindo-se a área foliar, utilizando um medidor de área foliar automático, marca Hayashi Denkoh, modelo AAM-7. Em seguida, o substrato foi lavado com água para a retirada das raízes. As folhas, o caule e as raízes foram secos em estufa de circulação forçada de ar a $65^{\circ} \mathrm{C}$, por 72 horas.

Para a obtenção dos teores foliares de nitrogênio no limão foi utilizada a metodologia semi-micro Kjeldahl, ou seja, digestão por ácido sulfúrico seguida por destilação e titulação. Para a determinação do teor de fósforo, foi utilizado o mé to do do ácido fosfovanadato molíbdico, descrito por Sarruge \& Haag (1974). Para a determinação do potássio, cálcio e magnésio, foi utilizado o método de espectofotometria de absorção atômica, descrito por Jorgensen (1977).

O experimento foi conduzido de acordo com o delineamento experimental inteiramente casualizado, com três tratamentos em oi to repetições.

Foram realizadas coletas da solução de solo de todos os vasos aos 45 e 58 dias após o transplante das mudas. As soluções obtidas através das cápsulas porosas foram recolhidas em garrafas de vidro hermeticamente fechadas. Para avaliar seus atributos físicos e químicos foram determinados: potencial osmótico (osmômetro de pressão de vapor, marca Wescor, modelo 5500), pH (pHmetro, marca Digimed, modelo DMpH-1), e condutividade elétrica (condutivímetro, marca Cyberbcan, modelo CON-100). Para quantificar os te ore s de nutrientes na solução foi utilizada a mesma metodologia empregada na análise foliar, modificada apenas na não digestão das amostras .

Os resultados foram submetidos a análise de variância pelo Teste $\mathrm{F}$ e a comparação das médias dos tratamentos foi feita pelo teste "t de Student" ao nível de 5\% de probabilidade. 


\section{RESULTADOS E DISCUSSÃO}

As mudas de limão responderam à adição de matéria seca de B. decumbens ao solo na concentração de $3 \%$, com red uções de $44 \%$ na altura, $42 \%$ no teor de clorofila, $34 \%$ no número de folhas, $57 \%$ na área foliar e $55 \%$ na biomassa seca total (Tabela 2 e Figuras 1 e 2).

TABELA 2. Efeitos de diferentes concentrações de $B$. decumbens no substrato sobre a a altura, diâmetro do caule, teor de clorofila total, número de folhas, área foliar e na massa seca de plantas de limão cravo, aos 58 dias após o transplante das mudas. Média de oito repetições.

\begin{tabular}{|c|c|c|c|c|c|c|c|c|c|}
\hline \multirow{2}{*}{$\begin{array}{c}\text { Tratamentos } \\
\%(p / p)\end{array}$} & \multirow{2}{*}{$\begin{array}{l}\text { Altura } \\
\text { (cm) }\end{array}$} & \multirow{2}{*}{$\begin{array}{l}\text { Diâmetro } \\
\text { (cm) }\end{array}$} & \multirow{2}{*}{$\begin{array}{l}\text { Clorofila } \\
\left(\mathrm{mg} / \mathrm{dm}^{2}\right)\end{array}$} & \multirow{2}{*}{$\begin{array}{l}\text { Número de } \\
\text { folhas }\end{array}$} & \multirow{2}{*}{$\begin{array}{c}\text { Área foliar } \\
\qquad\left(\mathrm{cm}^{2}\right)\end{array}$} & \multicolumn{2}{|c|}{ Massa Seca } & \multicolumn{2}{|l|}{ (g/planta) } \\
\hline & & & & & & Raizes & Caule & Folhas & Total \\
\hline 0 & $16,24 \mathrm{a}^{1}$ & $0.23 \mathrm{a}$ & 5,87 a & 17,87 a & 92,62 a & $0,367 \mathrm{a}$ & $0,280 \mathrm{a}$ & 0,797 a & 1,445 a \\
\hline 1,5 & $16.50 \mathrm{a}$ & $0.22 \mathrm{a}$ & $5,70 \mathrm{a}$ & 16,63 a & 94.82 a & $0,368 \mathrm{a}$ & $0.317 \mathrm{a}$ & 0,888 a & 1,574 a \\
\hline 3,0 & $9,04 \mathrm{~b}$ & $0.20 \mathrm{a}$ & $3,37 \mathrm{~b}$ & $11,75 \mathrm{~b}$ & $39,59 \mathrm{~b}$ & $0,216 \mathrm{~b}$ & $0.145 \mathrm{~b}$ & $0.286 \mathrm{~b}$ & $0,647 \mathrm{~b}$ \\
\hline$-\frac{\operatorname{Pr}>\mathbf{F}}{----}$ & 0.02 & 38,47 & 0.01 & ------ & 0,01 & $\begin{array}{l}---- \\
0,63\end{array}$ & 0,10 & 0.01 & 0,02 \\
\hline $\mathbf{F}$ & 13.28 & 1,00 & 14,09 & 8,14 & 15,05 & 6,52 & 9,76 & 15,64 & 12,73 \\
\hline C.V. $(\%)$ & 23.59 & 20,82 & 21.13 & 20,82 & 30,13 & 30,60 & 33,19 & 35,29 & 32,54 \\
\hline
\end{tabular}

1 Médias seguidas da mesma letra. dentro da mesma coluna. não diferem entre si pelo teste t de Student ao nivel de $5 \%$ de probabilidade.

O conhecimento da composição química da solução do solo é importante para estimar a disponibilidade de nutrientes das plantas (Moraes \& Dynia, 1990; Bassoi, 1990) e determinar as propried ades fis ico-químic as do solo (Souza, 1994, Velini, 1995).

Foi observado que o teor de nitrogênio total na solução do solo reduziu significativamente quando incorporou-se a $B$. decumbens a $1,5 \%$ e 3,0\% (p/p) (Tabela 3). Em fun çã o de ss es resultados, su ge re-se du as hipóteses. A primeira hipótese é que o nitrogênio pode ter sido imobilizado durante a decomposição da matéria seca pelos microrganismos do solo. A segunda é que pode ter ocorrido inibição da nitrificação. A nitrificação é aparentemente realizada por dois gêneros de bactérias: as Nitrosomonas, as quais oxidam o amônio para nitrito, e as Nitrobacter que oxidam o nitrito a nitrato (Brady, 1974; Alexander, 1977). Segundo Rice (1984), existem evidências indicando que os al eloquímicos in ibem fortemente a nitrificação em muitos casos.

Apesar de não se ter detectado diferença significativa no teor de $\mathrm{N}$ total na solução do solo entre as concentrações de $1,5 \%$ e $3,0 \%$ de $B$. decumbens, a concentração desse reduziu de 1,4 para $0,5 \mathrm{mg} / \mathrm{I}$ ( Tabela 2) com o aumento na concentração de B. decumbens incorporada. Apenas nesse último caso é que o teor de $\mathrm{N}$ na solução do solo foi realmente limitante para o crescimento das plantas (Tabela 2). Neste caso, a absorção de $\mathrm{N}$ total pela planta foi quase 4 vezes menor que a testemunha, refletindo, inclusive, no teor de clorofila das folhas (Tabela 2). Por outro lado, Souza (1994) demonstrou que a incorporação de matéria seca de $B$. decumbens, nas mesmas proporções, não reduziu a concentração de nitrogênio da solução de solo, em um período de 39 dias após o transplante das mudas. 


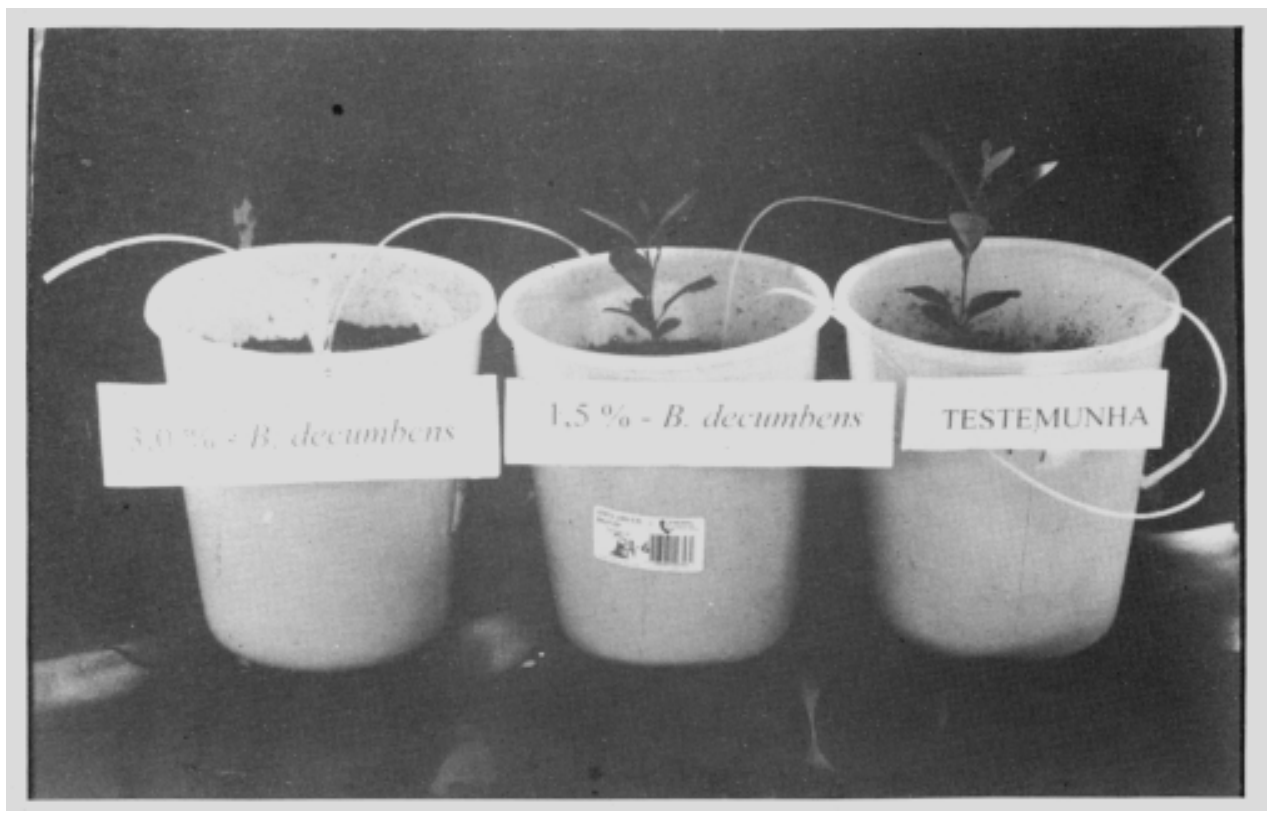

FIGURA 1. Efeito de diferentes proporções de $B$. decumbens sobre a parte aérea do porta-enxerto de limão cravo.

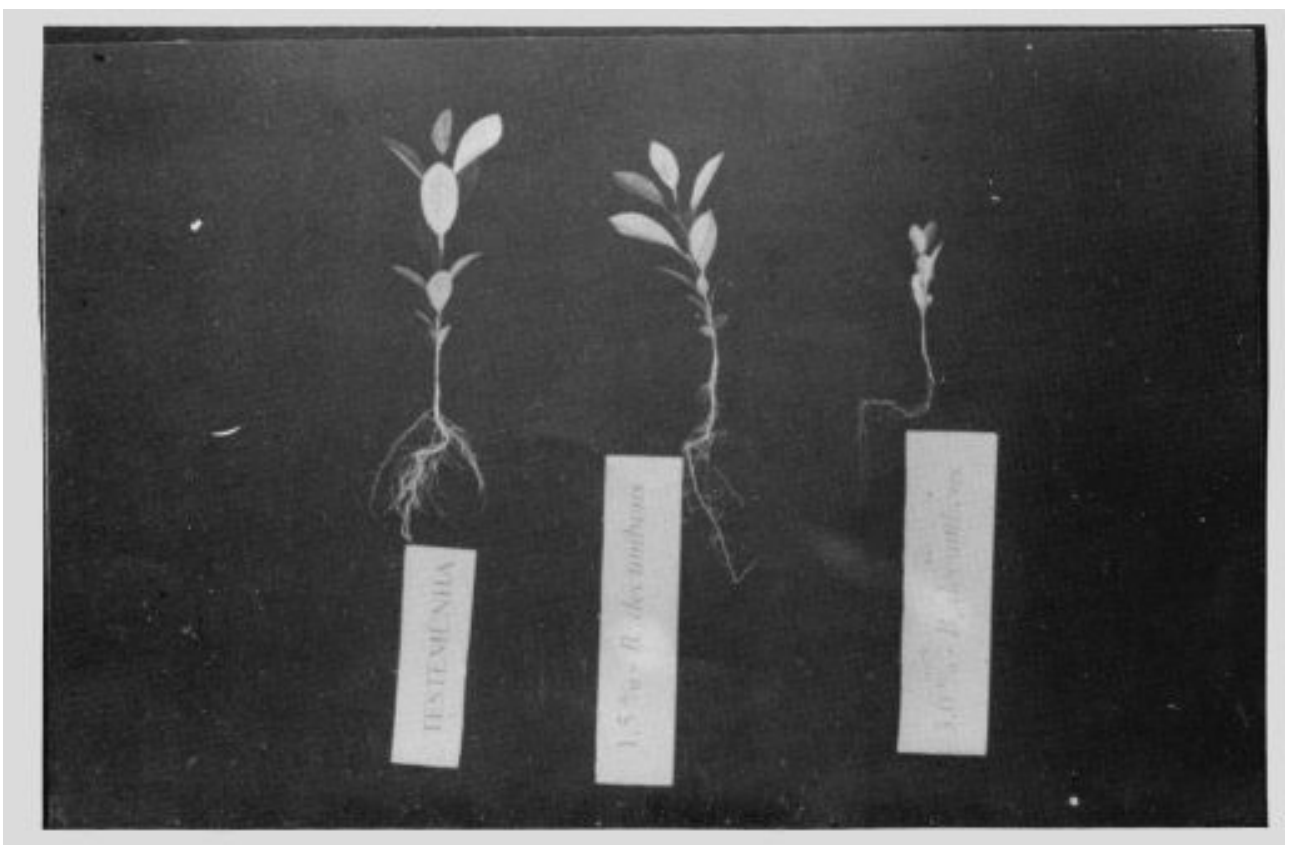

FIGURA 2. Efeito de diferentes proporções de $B$. decumbens sobre o sistema radicular do porta-enxerto de limão cravo. 
TABELA 3. Efeitos de diferentes concentrações de $B$. decumbens no substrato sobre os teores de macronutrientes da solução de solo (mg/I), aos 45 e 58 dias após o transplante das mudas. Média de oito repetições.

\begin{tabular}{|c|c|c|c|c|c|c|c|c|c|c|}
\hline \multirow{2}{*}{$\begin{array}{c}\text { Tratamentos } \\
\%(p / p) \\
\end{array}$} & \multicolumn{2}{|c|}{$\mathbf{N}$ (total) } & \multicolumn{2}{|c|}{$\mathbf{P}$} & \multicolumn{2}{|c|}{$\mathbf{K}$} & \multicolumn{2}{|c|}{$\mathrm{Ca}$} & \multicolumn{2}{|c|}{ Mg } \\
\hline & 45 dias & 58 dias & 45 dias & 58 dias & 45 dias & 58 dias & 45 dias & 58 dias & 45 dias & 58 dias \\
\hline 0 & $241.1 \mathrm{a}^{\prime}$ & $13,4 \mathrm{a}$ & $1,55 b$ & $0,33 \mathrm{~b}$ & $488.5 \mathrm{a}$ & $333,0 \mathrm{~b}$ & $817,0 \mathrm{a}$ & 839.5 a & $217,0 \mathrm{a}$ & $161,5 \mathrm{a}$ \\
\hline 1,5 & $1,4 \mathrm{~b}$ & $0,6 \mathrm{~b}$ & $2,13 b$ & $2,98 \mathrm{~b}$ & 499,5 a & 428,0 a & $782,0 \mathrm{a}$ & 924,5 a & 249,0 a & $180,0 \mathrm{a}$ \\
\hline 3,0 . & $\underline{0.5} \mathrm{~b}$ & $0.7 \mathrm{~b}$ & $7,13 \mathrm{a}$ & $5,11 \mathrm{a}$ & 486,0 a & 487,5 a & $\underline{542}, \underline{5} \mathrm{~b}$ & $900,0 \mathrm{a}$ & $199.5 \mathrm{a}$ & $175,0 \mathrm{a}$ \\
\hline $\operatorname{Pr}>\mathrm{F}$ & 0,01 & 0,01 & 1,86 & 0,08 & 96,06 & 0.74 & 3,31 & 69,76 & 17,49 & 62,91 \\
\hline $\mathrm{F}$ & 70.67 & 364,45 & 4,85 & 10,27 & 0,04 & 6,26 & 5,64 & 0,37 & 1,90 & 0,47 \\
\hline C.V $(\%)$ & 51,88 & 22,08 & 109,28 & 75,30 & 20,61 & 21,16 & 32,19 & 23,02 & 23,24 & 22,84 \\
\hline
\end{tabular}

1 Médias seguidas da mesma letra, dentro da mesma coluna, nào diferem entre si pelo teste t de Student ao nível de $5 \%$ de probabilidade.

É interessante observar que as concentrações dos outros macronutriente s (P, K, $\mathrm{Ca}$ e $\mathrm{Mg}$ ) na solução do solo, com a aplicação de $3 \%$ de $B$. decumbens, apresentaram médias iguais ou maiores que as da testemunha (Tabela 3), mostrando efeito de concentração, o que dá suporte à inferência de que houve efeito da incorporação de $B$. decumbens, na diminuição do teor de $\mathrm{N}$ no solo, e na sua absorção pela planta.

Os valores médios do potencial osmótico, pH e condutividade elétrica da solução do solo, estão apresentados na Tabela 4. Na análise de potencial osmótico houve uma redução significativa nos tratamentos com $B$. decumbens, $n a$ primeira avaliação (aos 45 dias), e na segunda (aos 58 dias), repetindo esse efeito significativo, somente na máxima concentração. Os resultados de potencial osmótico demonstraram que não houve retenção de água disponível nas mudas de limão, para os tratamentos com $B$. decumbens. Sabe-se que, uma alta retenção de água, é indicada quando apresenta abaixo de 3,00 -Bar (Almeida, 1993 ). Pelo contrário, verificou-se que a incorporação de matéria seca de $B$. decumbens, fez com que, os índices de potencial osmótico da solução fossem elevados em relação a testemunha.

TABELA 4. Efeitos de diferentes concentrações de B. decumbens no substrato sobre os atributos físicos e químicos da solução de solo, aos 45 e 58 dias após o transplante das mudas. Média de oito repetições.

\begin{tabular}{c|cc|cc|cc}
\hline $\begin{array}{c}\text { Tratamentos } \\
\%(\mathbf{p} / \mathbf{p})\end{array}$ & \multicolumn{2}{c|}{$\begin{array}{c}\text { Potencial osmótico } \\
\text { (-Bar) }\end{array}$} & \multicolumn{2}{c|}{$\mathbf{p H}$} & \multicolumn{2}{c}{$\begin{array}{c}\text { Condutividade elétrica } \\
\text { ( } \mathbf{\mu s} / \mathbf{c m})\end{array}$} \\
& $\mathbf{4 5}$ dias & $\mathbf{5 8}$ dias & $\mathbf{4 5}$ dias & $\mathbf{5 8}$ dias & $\mathbf{4 5}$ dias & $\mathbf{5 8}$ dias \\
\hline $\mathbf{0}$ & $3,27 \mathrm{a}$ & $2,45 \mathrm{a}$ & $5,7 \mathrm{~b}$ & $6,1 \mathrm{~b}$ & $680,4 \mathrm{a}$ & $546,0 \mathrm{a}$ \\
$\mathbf{1 , 5}$ & $2,66 \mathrm{~b}$ & $2,16 \mathrm{ab}$ & $5,9 \mathrm{~b}$ & $6,2 \mathrm{~b}$ & $536,4 \mathrm{~b}$ & $412,4 \mathrm{~b}$ \\
$\mathbf{3 , 0}$ & $2,41 \mathrm{~b}$ & $2,08 \mathrm{~b}$ & $7,4 \mathrm{a}$ & $7,6 \mathrm{a}$ & $425,4 \mathrm{~b}$ & $346,3 \mathrm{~b}$ \\
\hline $\mathbf{P r}>\mathbf{F}$ & 1,36 & 7,62 & 0,01 & 0,01 & 0,28 & 0,02 \\
$\mathbf{F}$ & 5,32 & 2,92 & 14,31 & 23,12 & 7,89 & 13,49 \\
$\mathbf{C . V} \mathbf{( \% )}$ & 18,62 & 13,41 & 10,90 & 7,46 & 23,52 & 18,02 \\
\hline
\end{tabular}

1 Médias seguidas da mesma letra, dentro da mesma coluna, não diferem entre si pelo teste t de Student ao nível de $5 \%$ de probabilidade. 
$\mathrm{O}$ pH da solução do solo manteve-se entre 5,9 e 7,6 para os tratamentos com $B$. decumbens, e na sua ausência entre 5,7 e 6,1 nas duas avaliações. O pH da solução do solo nos tratamentos com $B$. decumbens, permaneceram mais na faixa da neutralidade, para a máxima concentração, em relação a testemunha. Isto ocorreu, provavelmente, em decorrência do poder tampão do solo.

Os valores médios da condutividade elétrica (EC) dos tratamentos com $B$. decumbens ficaram entre 346,3 a $536,4 \mu \mathrm{s} / \mathrm{cm}$, sendo menores que os da testemunha (546,0 a 680,4 a $\mu \mathrm{s} / \mathrm{cm})$. No ge ral, águas com condudivida de elétrica inferiores a 750,0 is/cm são satisfatórias para a absorção pelas plantas (Klar, 1984). Pode-se considerar, que não ocorreu efeito de acumulação de sa is na solução do solo. À medida que aumentou a concen tração de $B$. decumbens no solo, diminuiu a condutividade elétrica na solução do solo, nas duas avaliações. Uma vez que, a conduti vidade elétrica é utilizada, comumente, para indicar os constituintes iônic os totai s da água, estando estreitamente relacionados à soma de cations (ou ânions), determinados quimicamente e com os sólidos dissolvidos (Klar, 1984).

As médias dos teores foliares de macro e micronutrientes (Tabela 5), em geral, encontramse na faixa considerada adequada, em relação a nutrição das plantas de limão (Malavolta et al., 1989), exce to para o nitrogênio, no tratamento onde a $B$. decumbens a $3 \%(\mathrm{p} / \mathrm{p})$ foi incorporada, cujo te or de $\mathrm{N}$ foi baix o. Provavelmente, em resposta a sua possível indisponibilidade no solo, demonstrado baixo, na solução do solo (Tabela 3).

TABELA 5. Efeitos de diferentes concentrações de $B$. decumbens no substrato sobre os teores foliares de macro $(\mathrm{g} / \mathrm{kg})$ e micronutrientes $(\mathrm{mg} / \mathrm{kg})$, aos 58 dias após o transplante das mudas. Média de oito repetições.

\begin{tabular}{|c|c|c|c|c|c|c|c|c|}
\hline $\begin{array}{c}\text { Tratamento } \\
\%(p / p)\end{array}$ & $\mathrm{N}$ & $\mathbf{P}$ & $\mathbf{K}$ & $\mathrm{Ca}$ & $\mathbf{M g}$ & $\mathrm{Cu}$ & Mn & $\mathrm{Fe}$ \\
\hline 0 & $26,0 \mathrm{a}^{1}$ & $0,9 \mathrm{~b}$ & $9,9 \mathrm{~b}$ & $9,0 \mathrm{a}$ & $1,6 \mathrm{a}$ & $40,0 \mathrm{a}$ & $232,5 \mathrm{a}$ & $680,0 \mathrm{a}$ \\
\hline 1,5 & $20,6 \mathrm{~b}$ & $1,1 \mathrm{~b}$ & $13,4 \mathrm{a}$ & $7,2 \mathrm{~b}$ & $1,3 \mathrm{~b}$ & $35,0 \mathrm{a}$ & $131,3 \mathrm{~b}$ & $363,8 \mathrm{~b}$ \\
\hline 3,0 & $15,2 \mathrm{~b}$ & $1,9 \mathrm{a}$ & $14,7 \mathrm{a}$ & $6,7 \mathrm{~b}$ & $1,5 \mathrm{ab}$ & $35,0 \mathrm{a}$ & $137,5 \mathrm{~b}$ & $395,0 \mathrm{ab}$ \\
\hline $\operatorname{Pr}>\mathrm{F}$ & 0,32 & 2,87 & 1,05 & 3,87 & 12,78 & 87,68 & 0,01 & 3,86 \\
\hline F & 7,79 & 4,41 & 6,02 & 3,96 & 2,33 & 0,13 & 18,69 & 3,96 \\
\hline C.V $(\%)$ & 21,85 & 24,09 & 20,76 & 20,49 & 22,00 & 57,52 & 20,56 & 48,07 \\
\hline
\end{tabular}

1 Médias seguidas da mesma letra, dentro da mesma coluna, nào diferem entre si pelo teste $\mathrm{t}$ de Student ao nível de $5 \%$ de probabilidade.

Nas condições em que o presente trabalho foi realizado, pode se concluir que:

A incorporação da matéria seca da parte aére a de $B$. decumbens, na concentração de $3 \%$ (p/p), inibiu o crescimento inicial de plantas de C. limonia, provavelmente por reduzir o teor de $\mathrm{N}$ na solução do solo e, consequentemente, na planta.

\section{LITERATURA CITADA}

ALEXANDER, M. Introduction to Soil Microbiology, 2. ed., New York, Wiley 1977.

ALMEIDA, M.A.M.P. Aspectos da germinaçã o de Phaseolus vulgaris L. cv. Carioca 80 $\mathrm{SH}$, em diferentes potenciais, água e salinidade. Botucatu: Faculdade de Ciências Agronomicas, Universidade 
Estadual Paulista 1993. 125p. Dissertação de Mestrado.

BASSOI, L.H. Lixiviação e acumulação de macronutrientes pelo (Zea mays L.) com e sem irrigação suplementar. Botucatu: FCA-UNESP, 1990, 94p. Dissertação de Mestrado.

BRADY, N.C. The Nature and Properties of Soil, 8.ed. Macmillan, New York, 1974.

BUCHHOLTZ, K.P. The influence of allelopathy on mineral nutrition. In: "Biochemical Interactions among Plants" (En viron. Physiol. Subcom., U.S . Natl. Comm. for IBP, eds), Natl. Acad. Sci., Washington, D.C., 1971. 86-89p.

HOSS NER, L.R., PHILLIPS,D.P. Extraction of soil solution from flooded soil using a porous plastic filter. Soil Sci., v.115, n.1, p.87-88, 1973.

JORGENSEN, S.S. Metodologia utilizada para análise químicas de rotina: guia prático. Piracicaba: Centro de Energia Nuclear na Agricultura, 1977.24p.

\section{KLAR, A.E. A Água no Sistema Solo-Planta-} Atmosfera. São Paulo, Nobel, 1984. 408p.

MALAVOLTA, E., VITTI, G.C., DE OLIVEIRA, S.A. Avaliação do Estado Nutricional das Plantas-Princípios e Aplicações. Piracicaba: Associação Brasileira para
Pesqui sa da Potassa e do Fosfato, 1989. 201p.

MORAES, J.F.V., DYNIA, J.F. Uso de cápsulas porosas para extrair solução do solo. Pesq. agropec. bras., v.25, n.10, p.1523-1528, 1990.

OLMSTED, C.E., RICE, E.L. Relative effects of known plant inhibitors on species from first two stages of old-field succession. Southwest. Nat., v.15, p.165-173, 1970.

RAID, B.V., QUAGGIO, J.A. Método de análise de solo para fins de fertilidade. Bol. Tec. Inst. Agron. (Campinas), n.81, 31p, 1983.

RICE, E. L. Alle lo pa thy, 2.ed. New York, Academic Press, 1984.363 p.

SARR UGE J.R., HAAG, H.P. Análise química em plantas. Piracicaba, Escola Superior de agricultura "Luiz de Queiroz", 1974. 56p.

SOUZA, L.S. Avaliação dos possíveis efeitos al elopátic os de diversas espécies de plant as daninhas sobre o crescime nto inicial de Eucalyptus grandis. Botucatu: FCA-UNESP. 1994. 104p. Dissertação de Mestrado.

VELINI, E.D. Evaluation of allelopathic effects of leguminous species, using leaf water extracts and soil solution. Botucatu: Departamento de Agricultura e Melhoramento Vegetal, Faculdade Paulista, 1995. 1-18p. 